\title{
Accurate Multiplexed Proteomics at the MS2 Level Using the Complement Reporter Ion Cluster
}

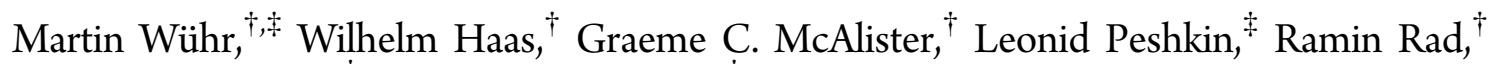 \\ Marc W. Kirschner, ${ }^{*}$ and Steven P. Gygi* ${ }^{\dagger}$ \\ ${ }^{\dagger}$ Department of Cell Biology, and ${ }^{\ddagger}$ Department of Systems Biology, Harvard Medical School, Harvard University, Boston, \\ Massachusetts 02115, United States
}

Supporting Information

\begin{abstract}
Isobaric labeling strategies, such as isobaric tags for relative and absolute quantitation (iTRAQ) or tandem mass tags (TMT), have promised to dramatically increase the power of quantitative proteomics. However, when applied to complex mixtures, both the accuracy and precision are undermined by interfering peptide ions that coisolate and cofragment with the target peptide. Additional gas-phase isolation steps, such as proton-transfer ion-ion reactions (PTR) or higher-order MS3 scans, can almost completely eliminate this problem. Unfortunately, these methods come at the expense of decreased acquisition speed and sensitivity. Here we present a method that allows accurate quantification of TMT-labeled peptides at the MS2 level without additional ion purification. Quantification is based on the fragment ion cluster that carries most of the TMT mass balance. In contrast to the use of low $\mathrm{m} / \mathrm{z}$ reporter ions, the localization of these complement TMT $\left(\mathrm{TMT}^{\mathrm{C}}\right)$ ions in the spectrum is precursor-specific; coeluting peptides do not generally affect the measurement of the $\mathrm{TMT}^{\mathrm{C}}$ ion cluster of interest. Unlike the PTR or MS3 strategies, this method can be implemented on a wide range of high-resolution mass spectrometers like the quadrupole Orbitrap instruments (QExactive). A current limitation of the method is that the efficiency of $\mathrm{TMT}^{\mathrm{C}}$ ion formation is affected by both peptide sequence and peptide ion charge state; we discuss potential routes to overcome this problem. Finally, we show that the complement reporter ion approach allows parallelization of multiplexed quantification and therefore holds the potential to multiply the number of distinct peptides that can be quantified in a given time frame.
\end{abstract}

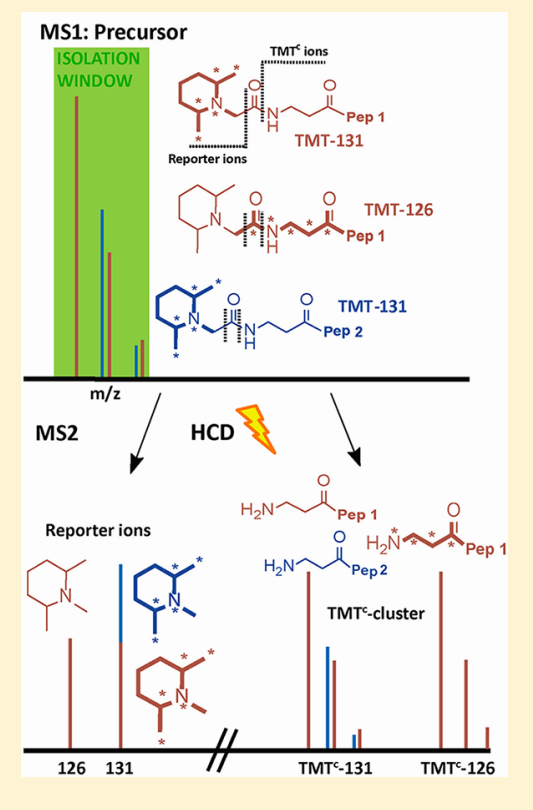

$\mathrm{M}$ ass spectrometry (MS)-based proteomics has undergone remarkable improvements over the past few years, resulting today in the identification of more than 10000 proteins from mammalian samples in a single experiment. ${ }^{1}$ While protein identification is now mature, accurate quantification among multiple conditions remains a challenge. Unpredictable ionization efficiencies currently prevent absolute quantification of protein abundance in high-throughput experiments. To avoid this limitation, methods have been developed where peptides from different conditions can be isotopically labeled so that their chemical structure is identical but their isotopic composition differs. When analyzed by MS, the relative change in protein abundance can be inferred from the relative abundance of the ions, which are unique to the different conditions investigated. ${ }^{2}$

Most commonly, peptides are quantified from MS1 spectra, based on relative abundance of differentially labeled species. One example is metabolic labeling. ${ }^{3}$ Alternatively, peptides from proteins obtained from different experimental conditions can be chemically modified to incorporate stable isotopes for quantification. ${ }^{4} \mathrm{~A}$ major disadvantage of these MS1-based quantification methods is that the complexity of the MS1 spectrum increases with the number of differentially modified peptides so that data acquisition speed and sensitivity is reduced due to redundant MS2 collection. ${ }^{5}$ While multiplexed proteomics with MS1-based quantification is feasible, e.g., reductive dimethylation with Lys-C-digested peptides allows the generation of five distinguishable species, the number of proteins that can be identified and quantified is reduced due to the increased complexity of the spectra. Hence, broad coverage of complex mixtures using MS1-based quantification is currently only used for two or maximally three conditions. ${ }^{6}$ Thus, there is a great need for a practical means of comparing a large number of samples in a single experiment, without sacrificing depth of coverage.

Isobaric tags, like tandem mass tags (TMT) or isobaric tags for relative and absolute quantitation (iTRAQ), are fundamentally different and promise multiplexed analyses with the potential for deep coverage. Peptides labeled with these tags have indistinguishable masses in the MS1 spectrum, thus not

Received: July 12, 2012

Accepted: September 18, 2012

Published: October 25, 2012 
increasing the complexity of the spectrum, but after fragmentation each component of a multiplexed sample produces a reporter ion with unique mass in the low $m / z$ region, which can be used for relative quantification. Presently up to eight channels have been commercialized. ${ }^{7}$ Isobaric labeling can be combined with traditional MS1-based quantification to increase the multiplexing capacity. An 18plex experiment has been demonstrated. ${ }^{8}$

It has become increasingly clear that there is a serious shortcoming of isobaric labeling. When analyzing complex mixtures, peptides selected for fragmentation are typically contaminated by coeluting ions of lower abundance. Reporter ions therefore originate from both target and interfering ions, causing a distortion of the quantification ${ }^{9}$ (Figure 1). Two

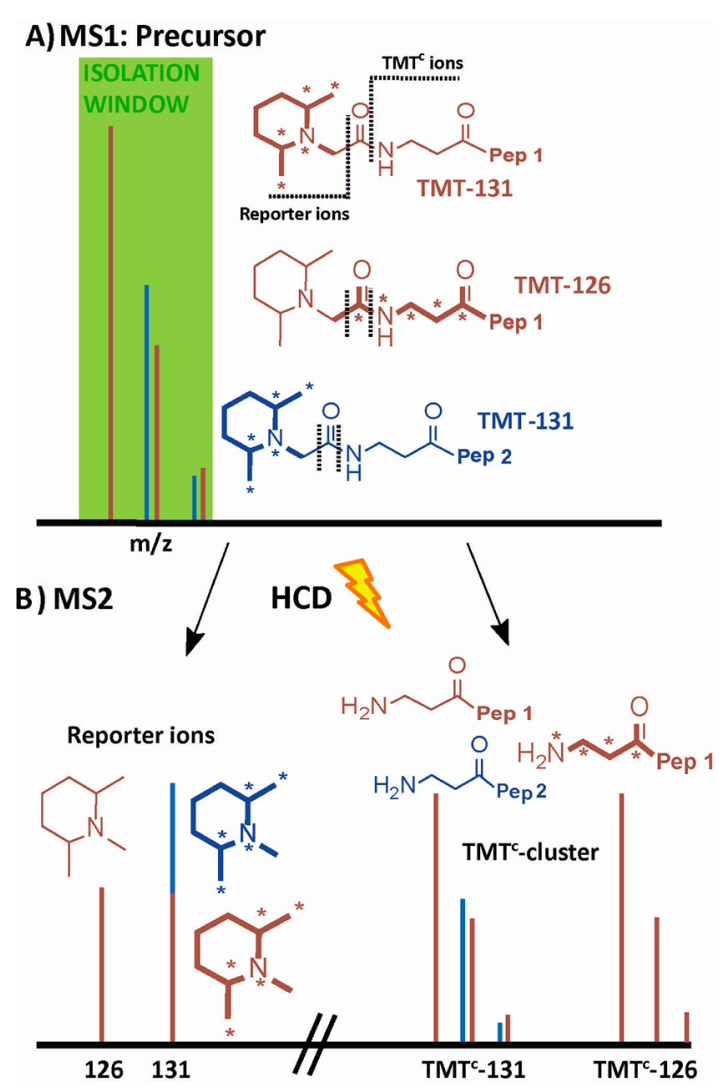

Figure 1. Principle of interference-free quantification based on the $\mathrm{TMT}^{\mathrm{C}}$ cluster. (A) A peptide (brown) is labeled with TMT-131 or TMT-126 reagents, and both forms are mixed in a ratio of $1: 1$. Asterisks on the TMT structures indicate heavy isotopes $\left({ }^{13} \mathrm{C}\right.$ or $\left.{ }^{15} \mathrm{~N}\right)$. An interfering peptide (blue) is coeluting with the peptide of interest within the isolation window for the MS2 spectrum. When peptide ions are subjected to higher-energy collisional dissociation (HCD), the TMT tags fragments at two positions, as indicated with dotted lines. (B) Typically, the MS2 spectrum contains both the reporter ions (left) and the $\mathrm{TMT}^{\mathrm{C}}$ cluster (right), which contains most of the massbalancing part of the TMT tag. Quantification based on the reporter ions is inaccurate as interfering peptides produce reporter ions of identical mass. In contrast with high-resolution MS2 spectra, the $\mathrm{TMT}^{\mathrm{C}}$ ions for the peptide of interest (and any coeluting peptides) can typically be distinguished, and therefore the $\mathrm{TMT}^{\mathrm{C}}$ ion cluster comprises accurate quantitative information. Note that this approach can be used to quantify multiple peptides in a single MS2 spectrum; e.g., in the toy example shown here the brown peptide ratio for the TMT-126 and TMT-131 channels would be 1:1, whereas the blue peptide ratio would be $0: 1$. strategies have been introduced to overcome this problem. Ting et al. reisolated the most abundant ion in the MS2 spectrum and refragmented it. The resulting reporter ions in the MS3 spectrum were then almost exclusively derived from the target peptide. $^{9 a}$ Alternatively, Wenger et al. reduced the charge state of the precursor peptide using proton-transfer ion-ion reactions (PTR) prior to fragmentation, thereby removing interfering ions with different charge states. ${ }^{9 \mathrm{~b}}$ While both methods drastically improve the accuracy and precision of quantification, they come at the cost of decreased data acquisition speed and sensitivity.

Here, we introduce an alternative approach for accurate isobaric quantification. It does not require an additional purification step; rather it exploits the high mass accuracy and resolution of modern mass spectrometers, including Orbitrap, FT-IR, and time-of-flight (TOF) instruments. As an alternative to using the reporter ions in the low $m / z$ region of the MS2 spectrum (TMT reporter ions), we quantify sample differences based on the complement TMT fragment ion cluster $\left(\mathrm{TMT}^{\mathrm{C}}\right.$ cluster), which originates from partial loss of the TMT tag (Figure 1, Supporting Information Figure 1). $\mathrm{TMT}^{\mathrm{C}}$ clusters carry the equivalent quantitative information about the relative levels of the differentially labeled peptides as the low-mass reporter ions and are essentially their complement. The position of the $\mathrm{TMT}^{\mathrm{C}}$ cluster is charge state specific, and the mass accuracy of modern instruments can easily distinguish fragment ions that differ by less than $0.02 \mathrm{~m} / z$, sufficient for accurately quantifying the $\mathrm{TMT}^{\mathrm{C}}$ clusters. By analyzing mixtures of TMT-labeled yeast and human peptides with known but different mixing ratios, we show that this method generates accurate quantitative data unaffected by interfering peptide ions. Finally, we demonstrate that this method can quantify multiple distinct peptides in the same MS2 spectrum if they are cofragmented. This opens up the future possibility to parallelize quantification of isobarically labeled peptides, potentially multiplying the number of quantified peptides in a multiplexed proteomic experiment.

\section{RESULTS AND DISCUSSION}

Complement TMT Ion Cluster. Peptides labeled with any one of six different TMT channels are indistinguishable in the MS1 spectrum but can be quantified upon fragmentation based on their low $\mathrm{m} / z$ reporter ions (reporter ions). Upon closer inspection of MS2 spectra from TMT-labeled peptides, we observed another ion cluster that we assigned to peptide ions solely fragmented at a bond within the TMT tags (Figures 1 and $2 \mathrm{~B}$ ). These ions are generated by cleavage of the amide bond of the TMT label (Supporting Information Figure 1A). ${ }^{10}$ The leaving group typically takes a charge; hence, the $\mathrm{TMT}^{\mathrm{C}}$ product ions have one less charge than the precursor. We termed these fragment ions complement TMT $\left(\mathrm{TMT}^{\mathrm{C}}\right)$ ions. $\mathrm{TMT}^{\mathrm{C}}$ ions carry most of the mass-balancing group and, therefore, contain information about the relative differences of the labeled peptides. Because the labeled carbonyl carbon is part of the leaving group, the $\mathrm{TMT}^{\mathrm{C}}-130$ and $\mathrm{TMT}^{\mathrm{C}}-129$ ions are indistinguishable in our analysis (Supporting Information Figure 1A). These complementary ion clusters are more complicated than their related low-mass reporter ions because each also reflects the isotopic envelope of the labeled peptide. Hence the TMT $^{\mathrm{C}}$ ion clusters of neighboring TMT channels overlap. To obtain the relative peptide level ratios we essentially have to deconvolve the $\mathrm{TMT}^{\mathrm{C}}$ ion cluster with the isotopic envelope of the precursor-peptide ions (Supporting Informa- 
A)

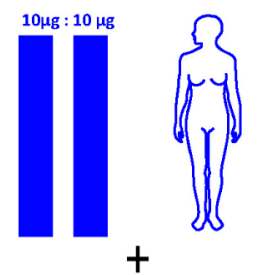

$1 \mu \mathrm{g}: 4 \mu \mathrm{g}: 10 \mu \mathrm{g}: 4 \mu \mathrm{g}: 1 \mu \mathrm{g}$

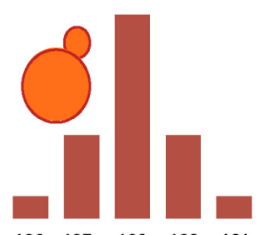

$\begin{array}{lllll}126 & 127 & 128 & 130 & 131\end{array}$

D)

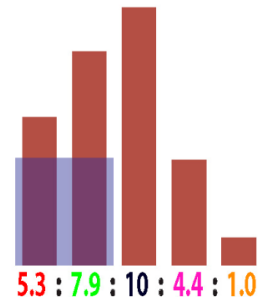

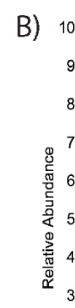

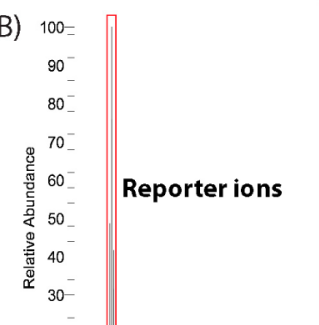

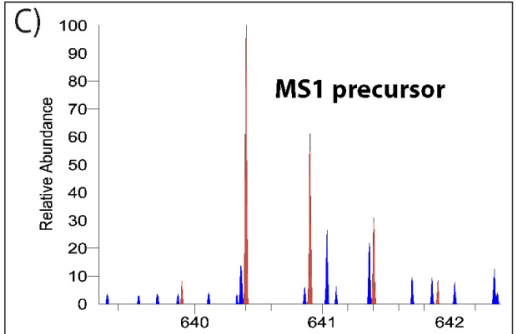
64
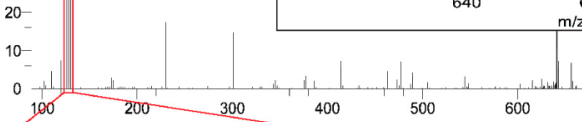

500

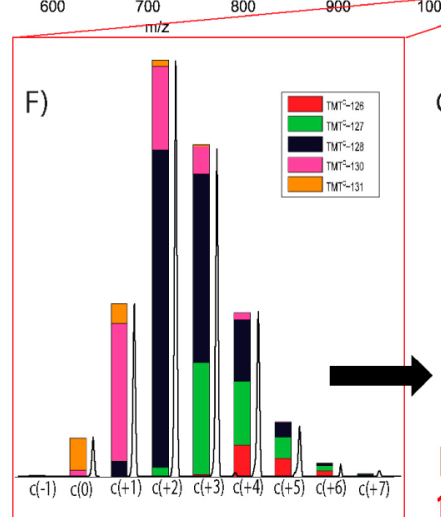

E)

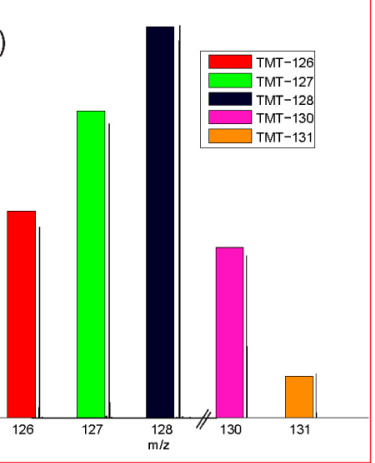

$\mathrm{TMT}^{\mathrm{c}}$ cluster

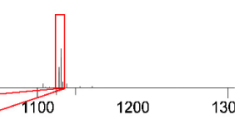

G)

$\begin{array}{lllll}126 & 127 & 128 & 130 & 131\end{array}$

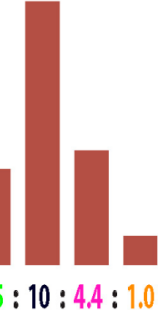

Figure 2. Comparison of TMT reporter ion and $\mathrm{TMT}^{\mathrm{C}}$ ion cluster quantification. (A) The human-yeast interference sample used throughout this study: peptides from human and yeast cells were digested with Lys-C and TMT-labeled. Amounts of $10 \mu \mathrm{g}$ (TMT-126) and 10 $\mu \mathrm{g}$ of TMT-127 of human peptides were combined with $1 \mu \mathrm{g}$ (TMT-126)/4 $\mu \mathrm{g}$ (TMT-127)/10 $\mu \mathrm{g}$ (TMT-128)/4 $\mu \mathrm{g}$ (TMT-130)/1 $\mu \mathrm{g}$ (TMT-131) of yeast peptides. Yeast peptide TMT ion ratio distortion through coisolated and fragmented human peptide ions is measured as interference. (B) MS2 spectrum of yeast peptide (AIELFTK) labeled with five-plex TMT from a run of human-yeast interference sample on a QExactive with 35k nominal resolution setting and $\pm 2 \mathrm{~m} / z$ isolation window. (C) Precursor ion cluster $\mathrm{m} / z$ range in the full MS spectrum. In this example interfering ions (blue) are visible next to the target precursor ion cluster (brown). (D) Reporter ion quantification is accurate in the interference-free channels (128-131), but a ratio distortion is observed in channels 126 and 127 due to interference of human peptide ions. (E) TMT reporter ion $\mathrm{m} / z$ region with colors representing contribution of each of the five channels. After correction of isotopic impurities we obtain the ratios shown in panel D. (F) TMT ${ }^{\mathrm{C}}$ ion cluster; $\mathrm{c}(0)$ indicates the position of the pseudomonoisotopic peak. Contributions of the five TMT channels (color coded) are optimized so that the summed square-differences between observed and theoretical $\mathrm{TMT}^{\mathrm{C}}$ clusters are minimal. $(\mathrm{G}) \mathrm{TMT}^{\mathrm{C}}$ ion cluster provides accurate yeast peptide quantification unaffected by interference.

tion Figure 1B). While it seems counterintuitive to use the $\mathrm{TMT}^{\mathrm{C}}$ cluster for quantification when the low $\mathrm{m} / z$ reporter ions quantitative information is easily obtainable, the TMT $^{\mathrm{C}}$ ions have the principle advantage that their unique location in the spectrum depends on the exact mass and charge of the tagged peptide (Figure 1B). In contrast, the small TMT reporter ions from both the target and any coisolated peptides are indistinguishable. We therefore reasoned that the peptidespecific TMT $^{\mathrm{C}}$ cluster would allow quantification at the MS2 level with negligible interference from coeluting peptides, avoiding the need of an additional gas-phase purification step.

Deconvolution of the $\mathrm{TMT}^{\mathrm{C}}$ Cluster in an MS2 Spectrum with Significant Interference. To evaluate the accuracy of quantification using the $\mathrm{TMT}^{\mathrm{C}}$ cluster, and in particular to test its susceptibility to interference, we created a sample of known mixing ratios in which we could identify and quantify the interference of coeluting peptides. ${ }^{9 a}$ We combined 1:4:10:4:1 $\mu \mathrm{g}$ of Lys-C-digested yeast peptides labeled with TMT in the channels $126,127,128,130$, and 131, respectively. To simulate interference, we added a mixture of $10: 10 \mu \mathrm{g}$ of human Lys-C-digested peptides labeled with TMT-126 and TMT-127, respectively (Figure 2A). We omitted the TMT-129 channel as the $\mathrm{TMT}^{\mathrm{C}}-129$ and $\mathrm{TMT}^{\mathrm{C}}-130$ ions are indistinguishable (Supporting Information Figure 1A). When we analyzed the interference sample using the traditional TMT reporter ions, we found that peptides exclusive to yeast were accurately quantified in the interference-free channels (128, 130 , and 131), but the relative abundance in the channels with human interference (126 and 127) were heavily distorted due to contaminating reporter ions of human origin (Figure 2D). In a real biological sample, where the mixing ratios would be unknown, we would be unable to distinguish which fraction of the reporter ions originated from the peptide of interest and which fraction originated from interfering coeluting peptides.

The MS2 spectrum shown in Figure $2 \mathrm{C}$ is from an experiment analyzed on a QExactive with $\pm 2 \mathrm{~m} / \mathrm{z}$ isolation width, a $90 \mathrm{~min}$ gradient, and $35 \mathrm{k}$ nominal resolution at 200 $m / z$. The spectrum identifies the yeast peptide AIELFTK. In the preceding MS1 spectrum, the precursor's isotopic envelope is marked in brown. Many other peaks (blue) are also visible within the isolation width for the MS2 analysis (Figure 2C). The TMT reporter ions are located in the low $m / z$ region of the MS2 spectrum. The spectrum's peak height and deduced relative abundance in Figure $2 \mathrm{E}$ do not completely agree as the intensity information is derived from the peak area, and we apply correction factors provided by the vendor to compensate for isotopic impurities. The $\mathrm{TMT}^{\mathrm{C}}$ cluster is located in the high $\mathrm{m} / z$ region of the spectrum (Figure 2, parts B and F). In this example the precursor ions carry two charges, while the $\mathrm{TMT}^{\mathrm{C}}$ ions are singly charged. Unlike the reporter ions, the position of 
A)

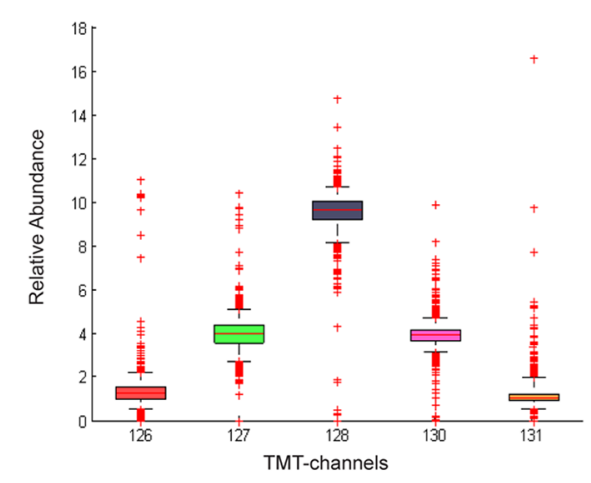

C)
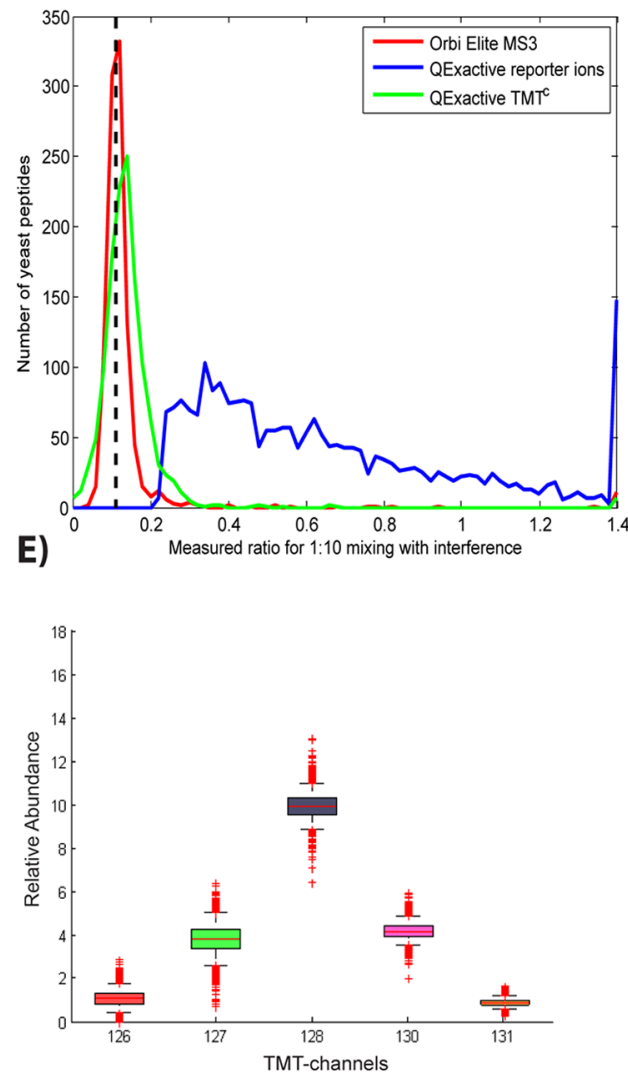

B)

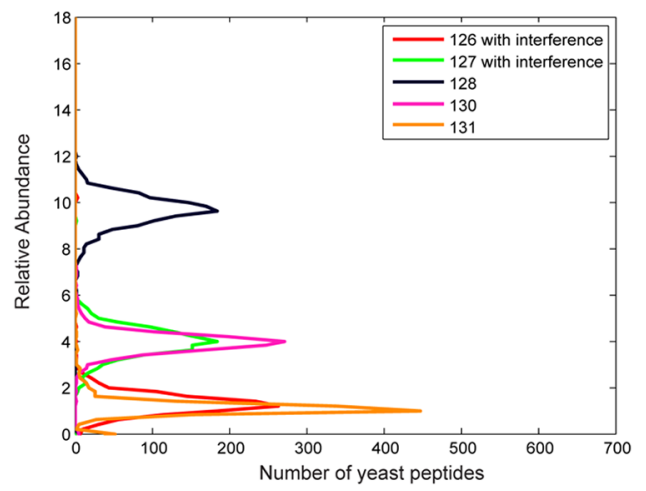

D)
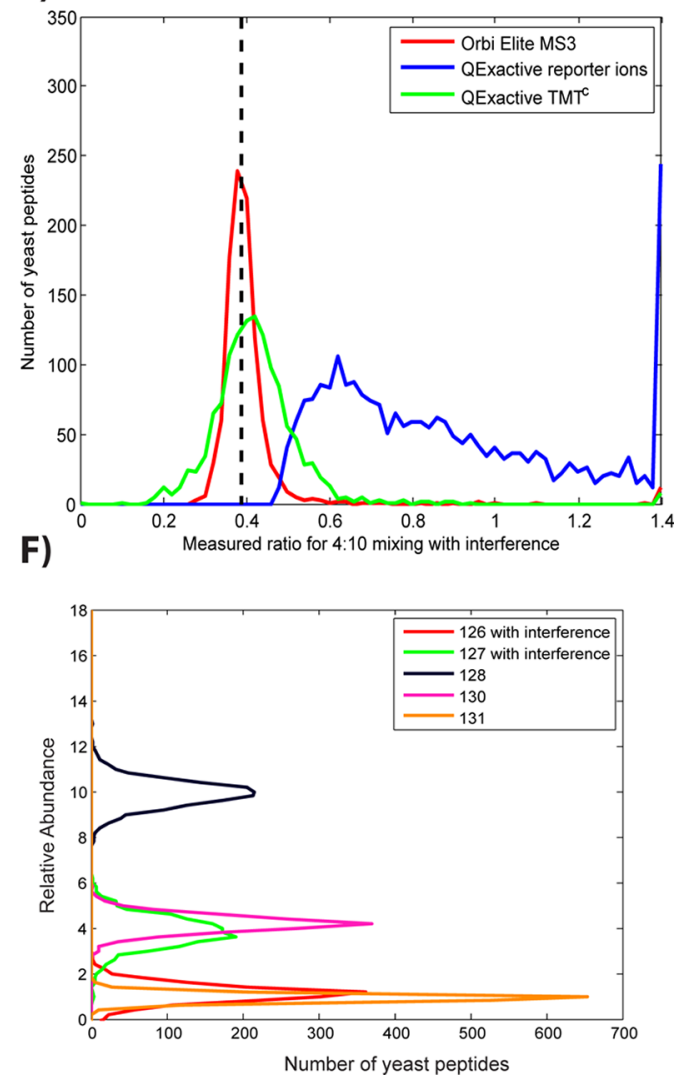

Figure 3. Large-scale evaluation of $\mathrm{TMT}^{\mathrm{C}}$ quantification. Panels A-D show yeast peptide ratios from the two-proteome human-yeast sample based on data acquired on a QExactive mass spectrometer. (A) Boxplot showing yeast peptide ratios determined using TMT ${ }^{\mathrm{C}}$ quantification (ratios are normalized to 20). Whiskers reach from the 5th to the 95th percentile. The median and numbers of outliers for channels with interference (126, $127)$ are very similar to equivalent channels without interference $(130,131)$. (B) Frequency distributions for ratios in panel A. Although interference does not seem to cause systematic errors, the ratio distribution for channels with interfering ions is wider than that for channels without interference. (C and D) Yeast peptides mixed in the ratios of 1:10 (TMT-126/TMT-128) and 4:10 (TMT-127/TMT-128), respectively, and subjected to interference by human peptides were quantified using TMT reporter ions (blue) or the TMT ${ }^{\mathrm{C}}$ approach (green). For evaluation, the same sample was also analyzed with an additional gas-phase isolation step (MS3) on an Orbitrap Elite (red). (E) Boxplot of Monte Carlo simulated yeast peptide ratios. The $\mathrm{TMT}^{\mathrm{C}}$ envelopes were simulated based on the known mixing ratios. As expected, the most striking difference to the actual experiment in panel A is the absence of extreme outliers. (F) Frequency distribution as in panel B of Monte Carlo simulated yeast peptide ratios. Although the extreme outliers are missing in the simulation the overall distribution is remarkably similar. As the simulation does not consider interfering peptides the wider distribution observed in the channels with interference is likely due to the increased overlap of the $\mathrm{TMT}_{126,127}^{\mathrm{C}}$ ion clusters with $\mathrm{TMT}^{\mathrm{C}}$ ions from other channels (see also Supporting Information Figure 4).

this ion cluster is dependent on the exact mass and charge state of the precursor. We define the peak of $\mathrm{TMT}^{\mathrm{C}}$ ions that are derived from the TMT-131 labeled pseudomonoisotopic precursor as the $\mathrm{c}(0)$ position and label all other peaks relative to this position. While in some spectra the $\mathrm{TMT}^{\mathrm{C}}$ clusters of coisolated peptides are easily observable (e.g., see Figure 5), often this is not the case. We believe this is due to interference originating from many different, low-abundant peptides, presumably often with different charge states, resulting in very low abundant and highly dispersed $\mathrm{TMT}^{\mathrm{C}}$ ions throughout 
the spectrum. We note that PTR experiments performed in the Coon lab demonstrated that isolation of ions with the charge state of the precursor peptide alone was sufficient to remove most interference. ${ }^{9 b}$

Deducing the original mixing ratios from the $\mathrm{TMT}^{\mathrm{C}}$ ion cluster is more complicated than deriving it from the low $\mathrm{m} / \mathrm{z}$ reporter ions. While the mass-balancing part of the TMT tag essentially encodes the relative quantitative information in the same way as the reporter ions, this information is convolved with the isotopic envelope of the labeled peptide. To deduce the original mixing ratio we essentially have to deconvolve the $\mathrm{TMT}^{\mathrm{C}}$ cluster with the isotopic envelope of the precursor peptide. Also, isotopic impurities from the TMT tags need to be considered (for detailed description of our calculation see the Materials and Methods and supplementary Figures 2 and 3 in the Supporting Information). The $\mathrm{TMT}^{\mathrm{C}}$ quantification in Figure 2 reports relative ratios of 1.0:3.5:10:4.4:1.0, which indicates similar ratios for channels with and without interference close to the known mixing ratios (Figure 2, parts $\mathrm{F}$ and $\mathrm{G}$ ). In contrast the reporter ion ratios are reported as 5.3:7.9:10:4.4:1.0 with a strong ratio distortion in the channels with interference (compare Figure 2, parts D and G).

Evaluating $\mathrm{TMT}^{\mathrm{C}}$ Quantification in a Complete Experiment. $\mathrm{TMT}^{\mathrm{C}}$ quantification across a complete experiment (of which Figure 2 is a sample) is shown in Figure 3. To evaluate the method, we plotted the median of the absolute deviation for the 1:10 and 4:10 channels with and without interference against the number of ions that we could observe in the TMT cluster (Supporting Information Figure 4A). For further analysis we excluded peptides of fewer than 1000 ions in the $\mathrm{TMT}^{\mathrm{C}}$ cluster. As an additional measure of quality, we evaluated how well the observed $\mathrm{TMT}^{\mathrm{C}}$ cluster fit the theoretical distribution (Supporting Information Figure 4B). Figure $3 \mathrm{~A}$ shows a boxplot of the filtered yeast peptides with ratios normalized to 20 . The whiskers reach from the 5 th to 95th percentile. Figure 3B depicts the corresponding histograms. While the 126 and 127 channels show a wider ratio distribution than the 130 and 131 channels, the medians for equivalent channels with and without interference are remarkably similar and very close to the known mixing ratios. Outliers seem to be fairly equally distributed among channels with and without interference. We will address the wider distribution for the channels with interference below. Taken together, the boxplot and histograms demonstrate that deconvolution of the $\mathrm{TMT}^{\mathrm{C}}$ ion cluster faithfully quantifies the isobarically labeled peptides in the MS2 spectrum, despite coeluting human peptides with different mixing ratios.

We also compared the performance of $\mathrm{TMT}^{\mathrm{C}}$ quantification with both the conventional MS2 reporter ion method and with the interference-free MS3 method. We quantified the yeast 1:10 and 4:10 ratios with interference $(126 / 128$ and $127 / 128)$ for $\mathrm{TMT}^{\mathrm{C}}$ and reporter ions on the QExactive (same experiment as described above) and compared it to the same sample analyzed on the Orbitrap Elite with the MS3 method and comparable elution gradient. The ratios obtained by the MS2 reporter ions were strongly distorted (Figure 3, parts C and D). By contrast, the $\mathrm{TMT}^{\mathrm{C}}$ derived medians for the $1: 10$ and 4:10 ratios with interference were centered close to the known mixing ratios with negligible distortion due to interference. The same was true for the ratios obtained with the MS3 method. While in this example we quantified $\sim 30 \%$ more peptides with the $\mathrm{TMT}^{\mathrm{C}}$ approach compared to the MS3 method (Table 1); the ratio distribution is notably wider for $\mathrm{TMT}^{\mathrm{C}}$, especially for the $4: 10$ ratio (Figure 3D).

Table 1. Number Comparison of Five-Plex Interference Sample Quantified with the TMT ${ }^{\mathrm{C}}$ Approach or the MS3 Method

\begin{tabular}{|c|c|c|c|}
\hline & $\begin{array}{l}\text { QExactive } \\
\text { TMT }^{\mathrm{C}}\end{array}$ & $\begin{array}{l}\text { Orbi Elite } \\
\text { TMT }^{\mathrm{C}}\end{array}$ & $\begin{array}{l}\text { Orbi } \\
\text { Elite } \\
\text { MS3 }\end{array}$ \\
\hline acquired MS2 spectra & 22024 & 10173 & 8843 \\
\hline acquired MS3 spectra & & & 7494 \\
\hline identified peptides $^{a}$ & 9390 & 4511 & 4063 \\
\hline identified yeast peptides & 4029 & 2046 & 1879 \\
\hline $\begin{array}{l}\text { identified yeast peptides with sufficient } \\
\text { ions for quantification }{ }^{b}\end{array}$ & 1567 & 1039 & 1024 \\
\hline $\begin{array}{l}\text { filter for agreement bw predicted and } \\
\text { observed } \text { TMT }^{\mathrm{C}} \text { cluster }^{c}\end{array}$ & 1291 & 924 & 1024 \\
\hline \multicolumn{4}{|c|}{$\begin{array}{l}{ }^{a} 1 \% \text { false discovery rate on peptide level. }{ }^{b} \text { At least } 1000 \mathrm{TMT}^{\mathrm{C}} \text { ions or } \\
\text { at least } 500 \text { reporter ions for the MS3 method. }{ }^{c} \text { Applies only for } \\
\mathrm{TMT}^{\mathrm{C}} \text { quantification; minDiff }<0.005 \text {. (See Materials and Methods } \\
\text { for detail.) }\end{array}$} \\
\hline
\end{tabular}

To evaluate the theoretical limit of the precision of the $\mathrm{TMT}^{\mathrm{C}}$ quantification, we simulated experimental sampling error for the number of ions observed in a Monte Carlo calculation, ignoring interference and other measurement errors. The resulting median absolute deviations of the simulated and measured ratios were remarkably similar (Supporting Information Figure 4, parts A and E). As expected, the simulated experiment was free of extreme outliers (Supporting Information Figure 4F). Interestingly, the boxplot and histograms of the simulated experiment showed a wider distribution of 126 and 127 channels compared to the 130 and 131 channels (Figure 3, parts E and F). We originally attributed this widening that we also observed in the actual experiment to interference. But the simulation is interference-free. We therefore conclude that the wider distribution in the lower TMT channels is likely due to the burying of the $\mathrm{TMT}_{126,127}^{\mathrm{C}}$ ions in the $\mathrm{TMT}^{\mathrm{C}}$ cluster (Figure $2 \mathrm{~F}$ ). As a result, measuring errors seem to accumulate and the precision of the measurement decreases. Overall, the simulated data were very similar to the real experiment, except for extreme outliers, suggesting a proximity to the theoretical limit of the present method.

Improving the Precision of the $\mathrm{TMT}^{\mathrm{C}}$ Method. We took advantage of the apparent agreement between actual experiment and Monte Carlo simulation (Figure 3, parts A, B, E, and $\mathrm{F}$ ) and tested if the precision of the $\mathrm{TMT}^{\mathrm{C}}$ quantification could be improved by a larger mass separation between the TMT channels. To this end, we simulated 10:10:10:10:10 ratios based on amino acid sequences and numbers of ions observed in the experiment described in Figure 3, parts A and B. We then analyzed the simulated $\mathrm{TMT}^{\mathrm{C}}$ cluster by the same method we used for the actual experiments. Supporting Information Figure 5A shows a boxplot of the obtained ratios. The precision of the middle channels $(127-130)$ is notably worse than the precision of the channels at the edges. When we removed the 128 channel the precision of all channels increases (Supporting Information Figure 5B). In contrast when removing only the 131 channel, there was less gain in precision (Supporting Information Figure 5C). This suggests that the improvement of precision is mostly due to the wider spacing of ions in the $\mathrm{TMT}^{\mathrm{C}}$ cluster rather than the higher number of ions per channel. A larger gain of precision could be achieved if each 


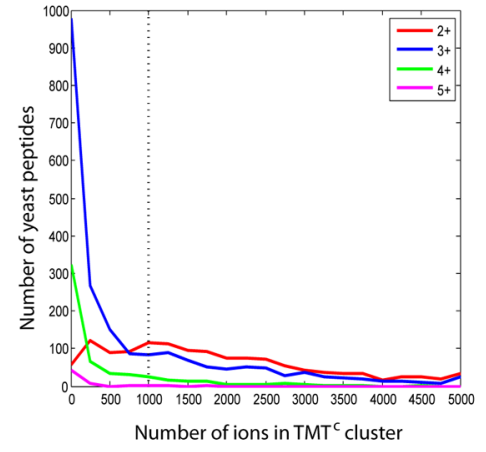

B)

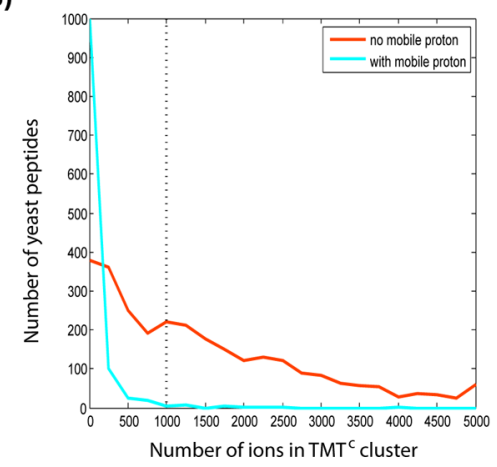

C)

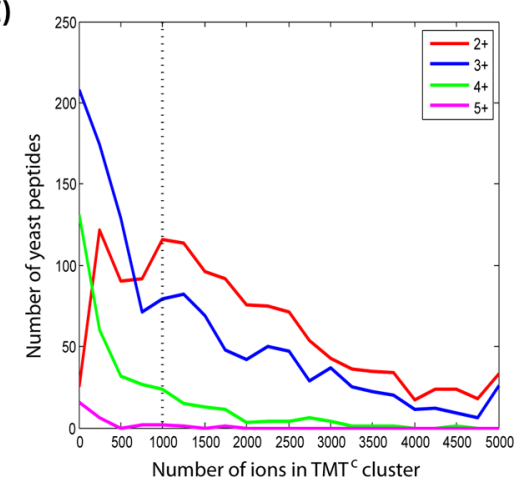

Figure 4. Amino acid sequence of peptides influences the $\mathrm{TMT}^{\mathrm{C}}$ ion cluster intensity. (A) Frequency distribution of the number of TMT ${ }^{\mathrm{C}}$ ions for different precursor charge states. A large fraction of higher charge state peptides does not produce significant amounts of TMT $\mathrm{T}^{\mathrm{C}}$ ions. The dotted line represents a 1000 ion cutoff as used throughout this study to filter quantitative data. (B) The differences observed in panel A can be partially explained by comparing peptides with (light blue) and without (orange) protons of high mobility, irrespective of charge state. Peptides with highmobility protons tend to yield insignificant numbers of TMT ${ }^{\mathrm{C}}$ ions. High-mobility protons likely support bond breakage at the peptide backbone and thereby suppress the formation of $\mathrm{TMT}^{\mathrm{C}}$ ions. (C) Frequency distributions of peptides not carrying a high-mobility proton for peptide ions of different charge states. The plot shows a negative correlation of peptide charge state and $\mathrm{TMT}^{\mathrm{C}}$ ion intensity. To some extent, this can be explained by the default MS instrument settings which prioritize precursors for MS2 spectra by the number of charges not ions. In addition, higher charge state peptides tend to be longer and might therefore be more likely to break at the peptide backbone, reducing the likelihood of $\mathrm{TMT}^{\mathrm{C}}$ ion formation.

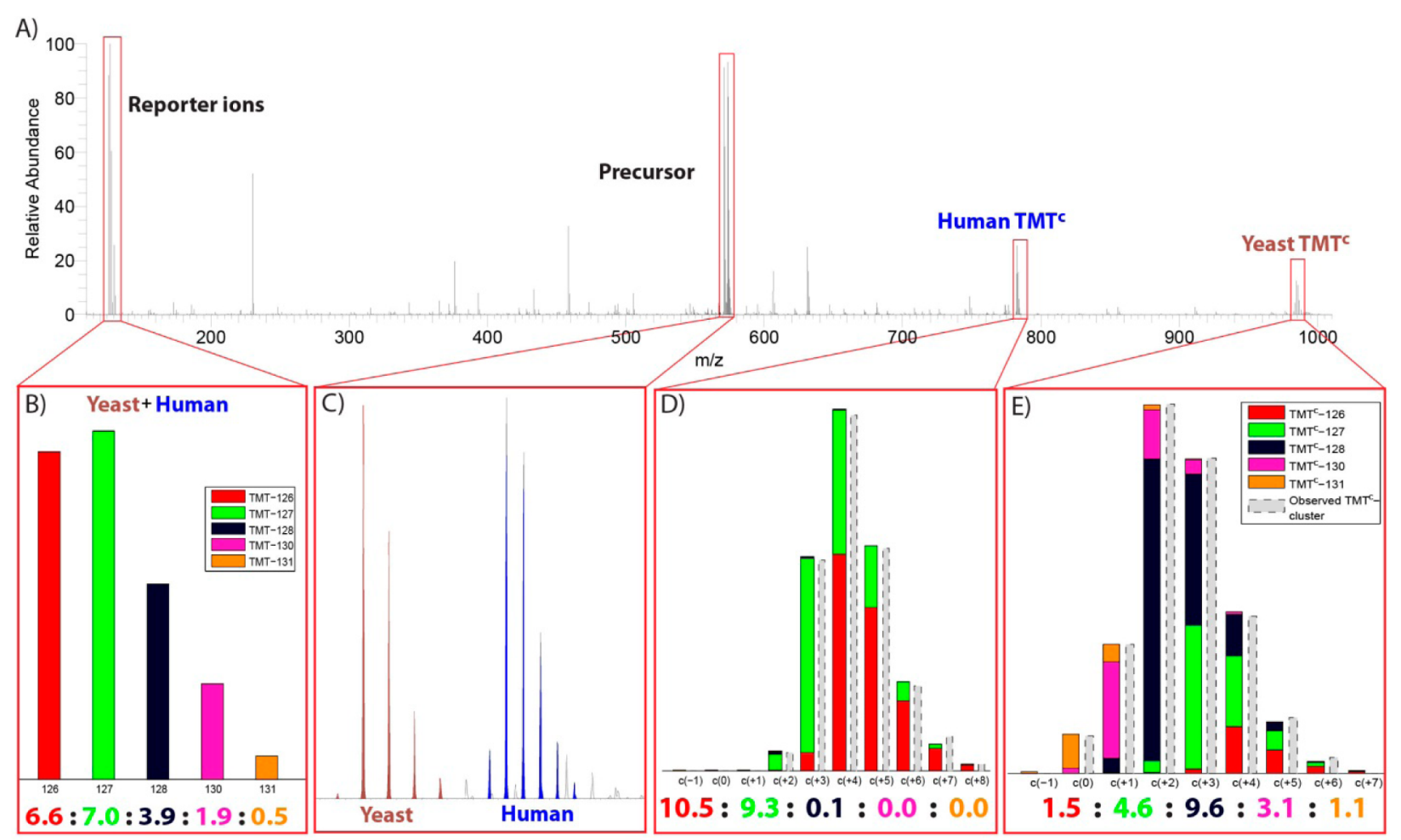

Figure 5. TMT ${ }^{\mathrm{C}}$ ion clusters allow quantification of multiple peptides from a single MS2 spectrum. (A) MS2 spectrum from an analysis of the twoproteome human-yeast sample applying a $\pm 3 \mathrm{~m} / z$ isolation window. (B) The ratios of the reporter ions (normalized to 20) indicate that peptide ions of both human and yeast origin were fragmented in this MS2 experiment. (C) Intact precursor ions of a doubly charged (brown) and triply charged (blue) peptide ions were detected. The peptide ions were identified as YTTLGK from yeast $(+2)$ and LDEREAGITEK from the human sample (+3). (D) The contribution of each TMT channel (colored) to the $\mathrm{TMT}^{\mathrm{C}}$ ion cluster of the human peptide is determined through deconvolution. (E) Equivalent representation of the $\mathrm{TMT}^{\mathrm{C}}$ ion cluster from the yeast peptide. For both panels $\mathrm{D}$ and $\mathrm{E}$ the ratio predictions based on deconvolution are close to the actual mixing ratios of the yeast and human peptides in the two-proteome sample.

channel were separated by at least $2 \mathrm{Da}$ (Supporting Information Figure 5D-H). We would like to note that the ion clusters which originate from the removal of multiple TMT reporter ions would have this desired property for a five-plex sample (data not shown). Unfortunately, for TMT-labeled peptides, these ion clusters form inefficiently.
Efficiency of $\mathrm{TMT}^{\mathrm{C}}$ Ion Formation. Table 1 summarizes the interference sample experiments run on the QExactive and Orbitrap Elite using the $\mathrm{TMT}^{\mathrm{C}}$ and MS3 quantification methods. Each analysis employed comparable elution gradients of $\sim 90 \mathrm{~min}$. Notably, the number of acquired MS2 spectra and the number of identified peptides on the QExactive-with 120 $\mathrm{ms}$ injection time and 35k nominal resolution-is nearly 
double the number of MS2 spectra acquired when an equivalent $\mathrm{TMT}^{\mathrm{C}}$ experiment was run on the Orbitrap Elite with only slightly higher nominal resolution (42k resolution, 50k AGC target, $250 \mathrm{~ms}$ maximum injection time). While the different experimental setups prevent an exact comparison, we believe that the different duty cycles are mostly due to the parallelization of ion injection and spectrum acquisition on the QExactive. In contrast, ion injection and spectrum acquisition on the Orbitrap Elite are sequential.

One of the advantages of the $\mathrm{TMT}^{\mathrm{C}}$ approach, when compared to the MS3 method, is that no additional purification step is necessary to provide interference-free quantification and a larger fraction of the precursor ion is potentially converted into (complement) reporter ions. This could either reduce the injection time for quantification and/or increase the sensitivity. However, with the current implementation the number of peptides that can be quantified in a given time are similar to the numbers obtained with the MS3 method (Table 1). ${ }^{9 a}$ This is mostly due to the insufficient formation of significant numbers of $\mathrm{TMT}^{\mathrm{C}}$ ions for a large fraction of peptides. When we separate the identified yeast peptide ions by their charge state, we observe that, with $120 \mathrm{~ms}$ injection time, upon fragmentation, $\sim 70 \%$ of doubly charged peptide ions create $\mathrm{TMT}^{\mathrm{C}}$ ions at an intensity that allows quantification (Figure 4A). This fraction decreases further for peptides with all charge states (Table 1). But it does not seem to be the charge state itself which results in lower efficiency of $\mathrm{TMT}^{\mathrm{C}}$ ion formation but a combination of charge state and amino acid composition. Likely peptide ions, which contain more charges than basic residues (arginine, lysine, histidine, and $\mathrm{N}$-terminus), exhibit at least one proton which is highly mobile. ${ }^{11}$ When we separate peptide ions based on this criterion we found that peptides with highly mobile protons generally do not generate $\mathrm{TMT}^{\mathrm{C}}$ ions at sufficient intensity. We believe that a highly mobile proton leads to an increased fragmentation at the peptide backbone thereby suppressing the formation of the $\mathrm{TMT}^{\mathrm{C}}$ ions. It has to be noted that even when considering peptide ions that do not carry a high-mobility proton, we still observe that peptide ions of higher charge states tend to form $\mathrm{TMT}^{\mathrm{C}}$ ions less efficiently (Figure 4C).

The inefficient TMT $^{\mathrm{C}}$ signal for a large fraction of peptides is the main limitation for $\mathrm{TMT}^{\mathrm{C}}$ quantification in its current implementation. While a significant problem, we believe there are viable solutions, with the chemistry of the isobaric tag as the most obvious target. The TMT tag was synthesized and optimized for the formation the low $\mathrm{m} / z$ reporter ions and not for $\mathrm{TMT}^{\mathrm{C}}$ ions. It should be possible to create a tag that forms the complement reporter ions more efficiently than the current TMT tag. For example, one could create a tag with a phosphoester bond. The neutral loss of the phospho group generally dominates the MS2 spectrum of phosphopeptides, especially with resonance collision-induced dissociation (CID) fragmentation. ${ }^{12}$ Furthermore, an additional basic group in the isobaric label might sequester high-mobility protons from the peptide backbone. More efficient formation of complement reporter ions should significantly increase the fraction of peptides amenable for quantification (Figure 4B) and should help the precision of the quantification for all peptides (Supporting Information Figure 5A).

$\mathrm{TMT}^{\mathrm{C}}$ Ion Cluster Facilitates Parallelization of Peptide Quantification. A unique advantage of the complement reporter ion approach over alternative quantification methods like MS3 or PTR is that the quantitative signal is entirely dependent on precursor characteristics. Inherently, this does not only lead to removal of interference but could allow the parallel quantification of coisolated peptides. In Figure 5, we provide a proof of principle for parallel quantification of multiple peptides in a single MS2 spectrum. By chance, we isolated two peptides for fragmentation during analysis of the human-yeast interference sample with $\pm 3 \mathrm{~m} / z$ isolation width (Figure 5A). Searches of the two precursors with Sequest against a human-yeast peptide database including decoys identified the yeast peptide YTTLGK for the +2 precursor and the human peptide LDEREAGITEK for the +3 precursor. As expected, reporter ions were accrued from both the yeast and human origin (Figure $5 \mathrm{~B}$ ). In contrast the $\mathrm{TMT}^{\mathrm{C}}$ clusters were unique to each peptide, and from these precursor-specific fragment ions the two peptides were quantified independently. The human peptide was quantified at 10.5:9.3:0.1:0.0:0.0, and the yeast peptide was quantified at 1.5:4.6:9.6:2.1:1.1 (ratios normalized to 20). We believe that the quantification of the yeast peptide suffered from the localization of the peptide precursor close to the edge of the isolation window. (The pseudomonoisotopic peak of the human peptide was the target, selected by the instrument, for the MS2 spectrum.) Therefore, the peaks at the lower $\mathrm{m} / \mathrm{z}$ side of the yeast isotopic envelope were less efficiently isolated, resulting in the overestimation of TMT-126 and TMT-127 channels. Even with this caveat, the quantifications for both peptides were reasonably close to the known, different mixing ratios, demonstrating that complement reporter ion quantification is uniquely applicable for methods where multiple precursors are intentionally isolated and fragmented like SWATH MS. ${ }^{13}$ Importantly, the wider isolation width does not seem to negatively affect the quantitation accuracy of the $\mathrm{TMT}^{\mathrm{C}}$ quantitation (Supporting Information Figure 6B). The data acquisition rate in multiplexed proteomic experiments is mostly limited by the ion injection time required for the accumulation of sufficient (complement) reporter ions in the MSn spectrum for quantification..$^{9 a}$ In comparison to these ion injection times the total acquisition time of MS2 spectra for identification is short. The complement reporter ion approach allows parallelizing the ion injection for accumulation of complement reporter ions, thereby opening up the opportunity to multiply the number of peptides that could be quantified in a given time frame.

\section{CONCLUSIONS}

Here we show that the complement reporter ion cluster $\left(\mathrm{TMT}^{\mathrm{C}}\right)$ can be used for accurate quantification of isobaric labeled peptides at the MS2 level. In its current implementation approximately half the peptides did not form sufficient $\mathrm{TMT}^{\mathrm{C}}$ ions to allow successful quantification. Nevertheless, the method was still competitive with existing methods and the acquired quantitative data was found to be almost completely unaffected by interfering peptide ions. We show routes to improve the complement reporter ion generation efficiency, to allow higher precision quantification of a larger number of peptides. Finally, we demonstrated that the complement reporter ion approach can be used to quantify multiple distinct peptides in a single MS2 spectrum. This has the potential to substantially increase the acquisition speed in multiplexed proteomics. 


\section{ASSOCIATED CONTENT}

\section{S Supporting Information}

Additional information as noted in text. This material is available free of charge via the Internet at http://pubs.acs.org.

\section{AUTHOR INFORMATION}

Notes

The authors declare no competing financial interest.

\section{ACKNOWLEDGMENTS}

We thank Lily Ting, Julian Mintseris, and Mike Springer for valuable advice and suggestions. This work was supported by NIH Grants GM026875 to M.W.K. and HG3456 and GM67945 to S.P.G.

\section{REFERENCES}

(1) (a) Nagaraj, N.; Wisniewski, J. R.; Geiger, T.; Cox, J.; Kircher, M.; Kelso, J.; Paabo, S.; Mann, M. Mol. Syst. Biol. 2011, 7, 548. (b) Beck, M.; Schmidt, A.; Malmstroem, J.; Claassen, M.; Ori, A.; Szymborska, A.; Herzog, F.; Rinner, O.; Ellenberg, J.; Aebersold, R. Mol. Syst. Biol. 2011, 7, 549. (c) Huttlin, E. L.; Jedrychowski, M. P.; Elias, J. E.; Goswami, T.; Rad, R.; Beausoleil, S. A.; Villen, J.; Haas, W.; Sowa, M. E.; Gygi, S. P. Cell 2010, 143, 1174-1189.

(2) Matthiesen, R.; Carvalho, A. S. Methods Mol. Biol. 2010, 593, 187-204.

(3) (a) Conrads, T. P.; Alving, K.; Veenstra, T. D.; Belov, M. E.; Anderson, G. A.; Anderson, D. J.; Lipton, M. S.; Pasa-Tolic, L.; Udseth, H. R.; Chrisler, W. B.; Thrall, B. D.; Smith, R. D. Anal. Chem. 2001, 73, 2132-2139. (b) Ong, S. E.; Blagoev, B.; Kratchmarova, I.; Kristensen, D. B.; Steen, H.; Pandey, A.; Mann, M. Mol. Cell. Proteomics 2002, 1, 376-386.

(4) (a) Tolonen, A. C.; Haas, W.; Chilaka, A. C.; Aach, J.; Gygi, S. P.; Church, G. M. Mol. Syst. Biol. 2011, 7, 461. (b) Gygi, S. P.; Rist, B.; Gerber, S. A.; Turecek, F.; Gelb, M. H.; Aebersold, R. Nat. Biotechnol. 1999, 17, 994-999.

(5) Mertins, P.; Udeshi, N. D.; Clauser, K. R.; Mani, D. R.; Patel, J.; Ong, S. E.; Jaffe, J. D.; Carr, S. A. Mol. Cell. Proteomics 2011, 11, M111.014423.

(6) (a) Deeb, S. J.; D’Souza, R. C.; Cox, J.; Schmidt-Supprian, M.; Mann, M. Mol. Cell. Proteomics 2012, 11, 77-89. (b) Olsen, J. V.; Vermeulen, M.; Santamaria, A.; Kumar, C.; Miller, M. L.; Jensen, L. J.; Gnad, F.; Cox, J.; Jensen, T. S.; Nigg, E. A.; Brunak, S.; Mann, M. Sci. Signaling 2010, 3, ra3.

(7) Ow, S. Y.; Cardona, T.; Taton, A.; Magnuson, A.; Lindblad, P.; Stensjo, K.; Wright, P. C. J. Proteome Res. 2008, 7, 1615-1628.

(8) Dephoure, N.; Gygi, S. P. Sci. Signaling 2012, 5, rs2.

(9) (a) Ting, L.; Rad, R.; Gygi, S. P.; Haas, W. Nat. Methods 2011, 8, 937-940. (b) Wenger, C. D.; Lee, M. V.; Hebert, A. S.; McAlister, G. C.; Phanstiel, D. H.; Westphall, M. S.; Coon, J. J. Nat. Methods 2011, 8, 933-935. (c) Ow, S. Y.; Salim, M.; Noirel, J.; Evans, C.; Rehman, I.; Wright, P. C. J. Proteome Res. 2009, 8, 5347-5355.

(10) Pichler, P.; Kocher, T.; Holzmann, J.; Mazanek, M.; Taus, T.; Ammerer, G.; Mechtler, K. Anal. Chem. 2010, 82, 6549-6558.

(11) Wysocki, V. H.; Tsaprailis, G.; Smith, L. L.; Breci, L. A. J. Mass Spectrom. 2000, 35, 1399-1406.

(12) Beausoleil, S. A.; Jedrychowski, M.; Schwartz, D.; Elias, J. E.; Villen, J.; Li, J.; Cohn, M. A.; Cantley, L. C.; Gygi, S. P. Proc. Natl. Acad. Sci. U.S.A. 2004, 101, 12130-12135.

(13) Gillet, L. C.; Navarro, P.; Tate, S.; Roest, H.; Selevsek, N.; Reiter, L.; Bonner, R.; Aebersold, R. Mol. Cell. Proteomics 2012, 11, O111.016717. 